\title{
Non-invasive assessment of vascular alteration using ultrasound
}

\author{
Woo-In Yang ${ }^{1}$ and Jong-Won $\mathrm{Ha}^{2,3^{*}}$
}

\begin{abstract}
It is well known that arterial function relates to cardiovascular morbidity and mortality. The noninvasive technology for the assessment of arterial function has developed as the importance of prevention of early cardiovascular disease has been emphasized. Over 2-D and Doppler echocardiography, speckle-tracking echocardiography has emerged as a valuable ultrasound imaging technique that allows for an objective and quantitative evaluation of global and regional myocardial function. Recently, several studies have extended its applicability beyond cardiac chambers, such as artery. Measurement of carotid arterial strain with speckle tracking ultrasound has been shown to be feasible and reliable. This review describes the new ultrasound techniques to assess arterial function and their clinical implications.
\end{abstract}

Keywords: Arterial function, Carotid artery, Ultrasound

\section{Background}

\section{Why do we need to evaluate arterial function?}

Cardiovascular disease (CVD) is the leading cause of morbidity and mortality in the industrialized countries [1]. In the early half of the 20th century, CVD increased rapidly as a result of industrialization and urbanization. However, during the latter half of the 20th century, the mortality of CVD decreased impressively due to advances in medical technology [1]. Coronary angiography has been the standard for the diagnosis and management of coronary artery disease for decades. However, physician's interests are going over to noninvasive assessment of vascular function as the importance of prevention of early CVD has been emphasized.

Previously, vascular aging, the changes of vascular function and structure, was overlooked in comparison with overt atherosclerosis. The cardiovascular continnum was restricted to atherosclerotic disease [2, 3]. However, vascular aging, in association with increased arterial stiffness, influences on the heart and other organs [4]. Vascular aging is correlated with systolic

\footnotetext{
* Correspondence: jwha@yuhs.ac

${ }^{2}$ Cardiology Division, Severance Cardiovascular Hospital, Yonsei University College of Medicine, Seoul, Republic of Korea

${ }^{3}$ Severance Biomedical Science Institute, Yonsei University College of Medicine, 134 Shinchon-dongSeodaemun-gu, Seoul 120-752, Republic of Korea

Full list of author information is available at the end of the article
}

hypertension, atherosclerosis, diastolic heart failure and small vessel disease in brain and kidney [4]. It is well known that arterial function relates to cardiovascular morbidity and mortality [5-7]. In the late 19th century, William Osler already underscored the importance of vascular aging by saying "you are as old as your arteries." Recently vascular aging has become one of the most important issue and regarded as the extended cardiovascular continnum [8]. These changes engage with the development of noninvasive technology for the assessment of arterial function.

This review describes the new ultrasound techniques to assess arterial function and their clinical implications.

\section{Basic pathophysiology of arterial function}

The arterial wall is mainly composed of scaffolding proteins, elastin and collagen [9]. Repetitive pulsation (approximately 30 million per year) results in damage of elastic lamellae and replacement with collagen in the arterial walls [10]. In various vasculopathy or with aging, elastin becomes broken and diminished in quantity [10]. Furthermore, the collagen matrix increases in a disorganized form [10]. These changes result in arterial dilatation and stiffening [4]. As arterial stiffness increases, flow pulsations cannot be buffered and are directly transmitted to distal arteries. This repeated mechanical stress damages arterial wall and results in vascular 
inflammation and atherosclerosis [11-13]. Generally, arterial pressure wave analysis and pulse wave velocity (PWV) have been regarded as valuable tools to evaluate arterial stiffness $[14,15]$. However, these methods assess global vascular changes rather than local alterations, which usually precede. Therefore, to detect early changes of vascular alteration, techniques that can evaluate local vascular changes would be quite useful, such as vascular ultrasound. Among the numerous arteries, common carotid artery is most frequently used for the assessment of arterial properties since it allows good spatial resolution when using ultrasound. During the arterial assessment, various parameters can be measured and carotid intima-media thickness (cIMT) has been suggested as a surrogate measure of vascular alterations, and several studies have shown that increasing CIMT is associated with subsequent coronary heart disease and stroke [16, 17].

Since it is non-invasive, reproducible, and simple, measurement of cIMT with ultrasonography is widely used to quantify the extent of subclinical disease in individuals with cardiovascular (CV) risk factors and to follow up in interventional studies. However, cIMT may not reflect the whole arterial changes occurring in various pathologic conditions. Before the progression of the intima and media thickening, the carotid artery may experience functional alterations, of which the occurrence is also associated with an increased risk of CV morbidity or mortality by augmenting arterial impedance and resultant increase in the afterload of the heart [18]. Recently, with advancements in ultrasound techniques, a novel

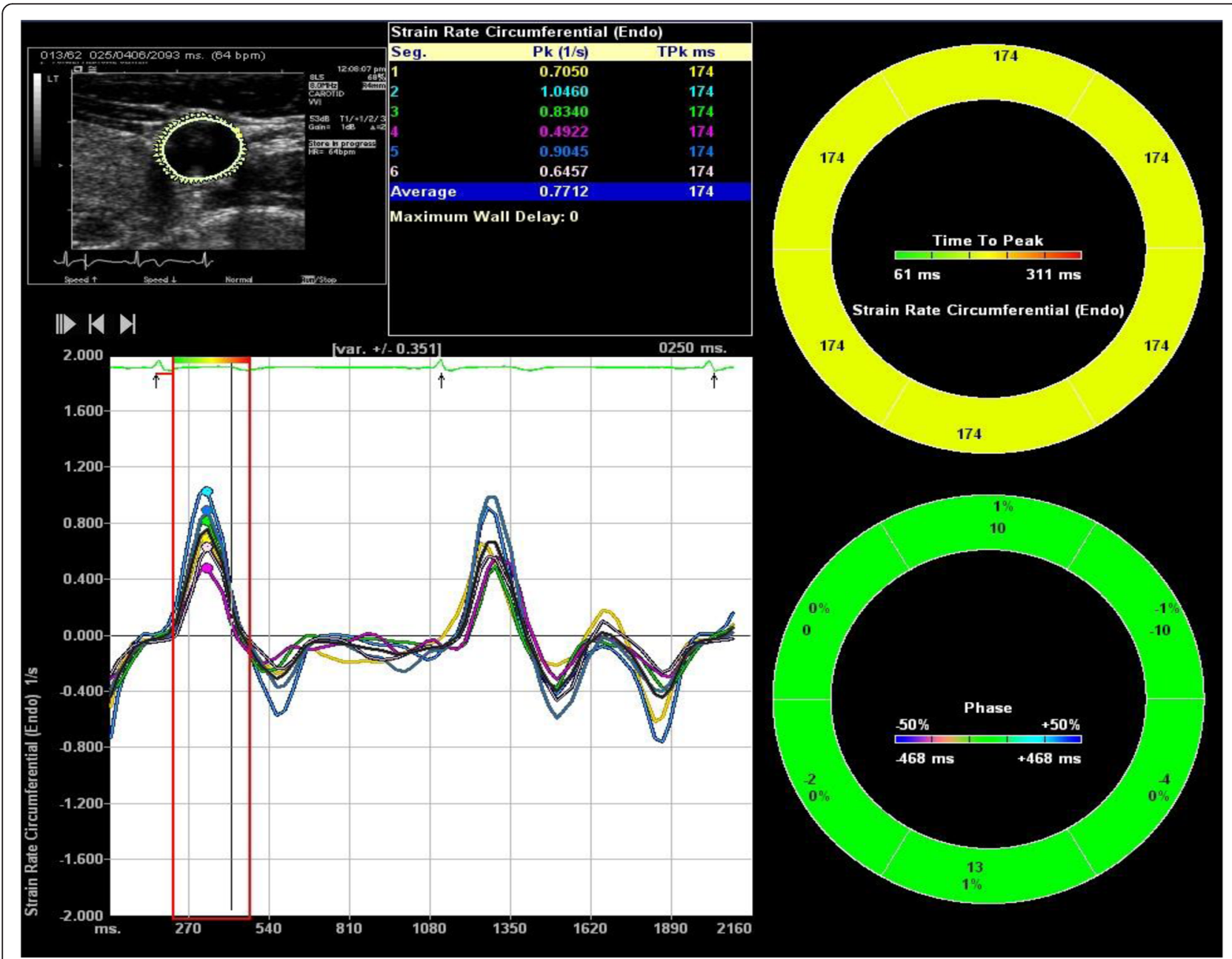

Fig. 1 The evaluation of arterial wall mechanics using Wl: The media-adventitia borders of carotid arteries were manually traced, and the peak circumferential strain rate and times to peak circumferential strain rate of six segments were analyzed automatically. The peak circumferential strain and the times to peak strain were similarly measured 
technique has facilitated the instantaneous quantification of carotid arterial mechanics.

\section{Novel technique in evaluation of vascular function; strain imaging}

Over 2-D and Doppler echocardiography, speckletracking echocardiography has emerged as a valuable ultrasound imaging technique that allows for an objective and quantitative evaluation of global and regional myocardial function $[19,20]$. Speckle-tracking echocardiography analyzes the spatial dislocation of speckles (defined as spots generated by the interaction between the ultrasound beam and myocardial fibers) on routine 2-D ultrasound imaging [21]. By tracking the displacement of speckles during the cardiac cycle, speckle-tracking echocardiography evaluates myocardial deformation. The 2-D speckle tracking method allows for angle-independent, rapid, and direct assessment of tissue motion and deformation [19, 20]. Based on this new technique, greater understanding into the pathophysiology of various cardiovascular diseases could be gained. Recently, several studies have extended its applicability beyond cardiac chambers, such as artery.

Measurement of carotid arterial strain with speckle tracking ultrasound has been shown to be feasible and reliable [5]. Recent studies reported that circumferential strain of common carotid artery was more sensitive in discrimination of aged artery and risk stratification, compared with cIMT and conventional 2-D ultrasound

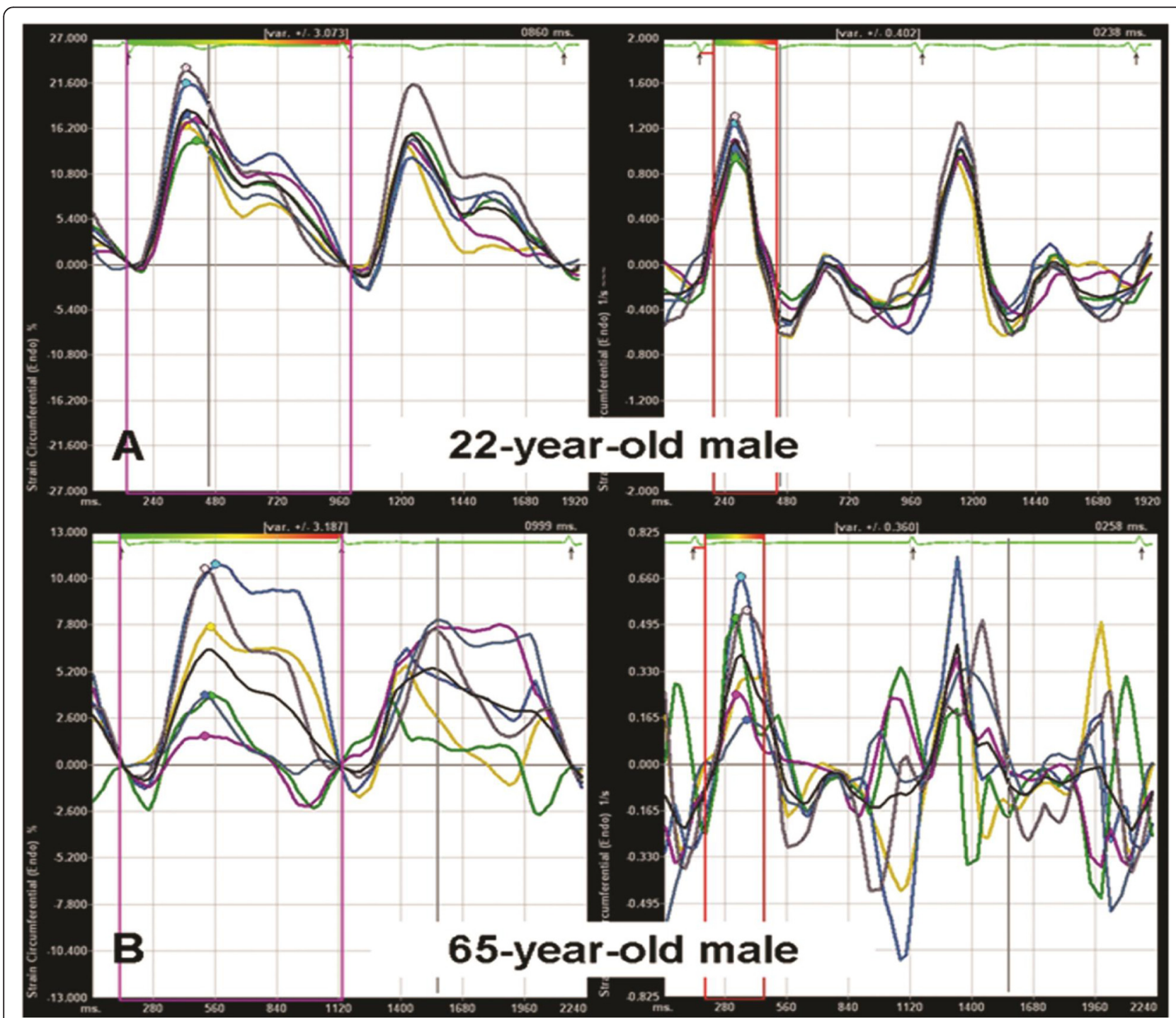

Fig. $2 \mathrm{Wl}$ analysis: circumferential strain and strain rate of six segments in a 22-year-old male subject (a) and a 65-year-old male subject (b). The carotid artery of the older subject showed a more reduced and asynchronous arterial expansion during systole. WI = velocity vector imaging (adapted from Ref 29) 
derived stiffness variables [22, 23]. In a study with coronary artery disease, carotid arterial strain was related with the presence and extent of coronary artery disease [24]. Svedlund et al. demonstrated that reduced longitudinal function of common carotid artery assessed by strain imaging predicted adverse cardiovascular event after 1 year [25].

Velocity vector imaging (VVI) is a new method of strain imaging with 2-D speckle tracking $[26,27]$. We have previously shown the feasibility of using VVI in the evaluation of arterial wall mechanics and have demonstrated the characteristics of arterial mechanics accompanying aging and vasculopathies using VVI (Fig. 1) [28-30]. Fractional area change and strain and strain rate by VVI were reduced according to age and significantly correlated with conventional parameters of arterial stiffness such as PWV and augmentation index (AIx) [29]. More importantly, VVI measurements of time to peak strain and strain rate enabled the assessment of the synchronicity of arterial systolic expansion. The SD of time to peak strain and strain rate significantly increased with age, suggesting non-uniform arterial expansion during systole with age (Fig. 2). PWV and AIx are considered as representative markers for vascular aging. However, PWV is increased more prominently in older age whereas AIx is a sensitive marker of arterial stiffening in relatively younger subjects [15]. Unlike PWV or AIx, asynchronicity of arterial systolic expansion rises steadily with aging and is independent of hemodynamic conditions including heart rate (Figs. 2 and 3) [29]. Therefore, asynchronous arterial expansion could be a more sensitive marker of vascular aging at all ages.

Arterial function using VVI was also evaluated in patients with vasculopathy such as Takayasu's arteritis and Marfan syndrome $[28,30]$. In Takayasu's arteritis and Marfan syndrome, arterial strain and strain rate were all reduced. Furthermore, SD of time to peak strain and strain rate were significantly increased, suggesting dyssynchronous arterial expansion during systole. According to the previous investigations, VVI has unique ability to provide an information regarding both regional and segmental alterations of arterial elastic properties in various conditions. In an animal study to assess the correlations between the VVI parameters and histologic changes, the radial velocity and circumferential strain had independent associations with the collagen content of the corresponding aortic wall [31].

Previously, most studies on arterial aging have focused on global changes of arterial function due to lack of test for regional arterial function. Conventional methods for assessment of arterial function usually represent global arterial function, and not regional function. Despite its potential importance, regional structural changes of artery and consequent aynchronicity of arterial expansion has been overlooked. Unlike other assessment technique for arterial function, strain analyses present regional arterial function. Therefore, assessment of vascular function with strain imaging could be expected to offer better understanding into the pathophysiology of vasculopathy.

Carotid 2-D ultrasound evaluates not only arterial structure but also arterial function including changes in diameter during the cardiac cycle [14]. However, these assessments are limited, in that they can be influenced by blood pressure and have less than optimal reproducibility [32, 33]. In the Rotterdam study, carotid distensibility as assessed by ultrasound failed to show any association with cardiovascular disease or mortality [6]. Even though there are not many studies, compared with ultrasonographic 2-D measures of arterial stiffness, arterial strain and strain rate are more sensitive for
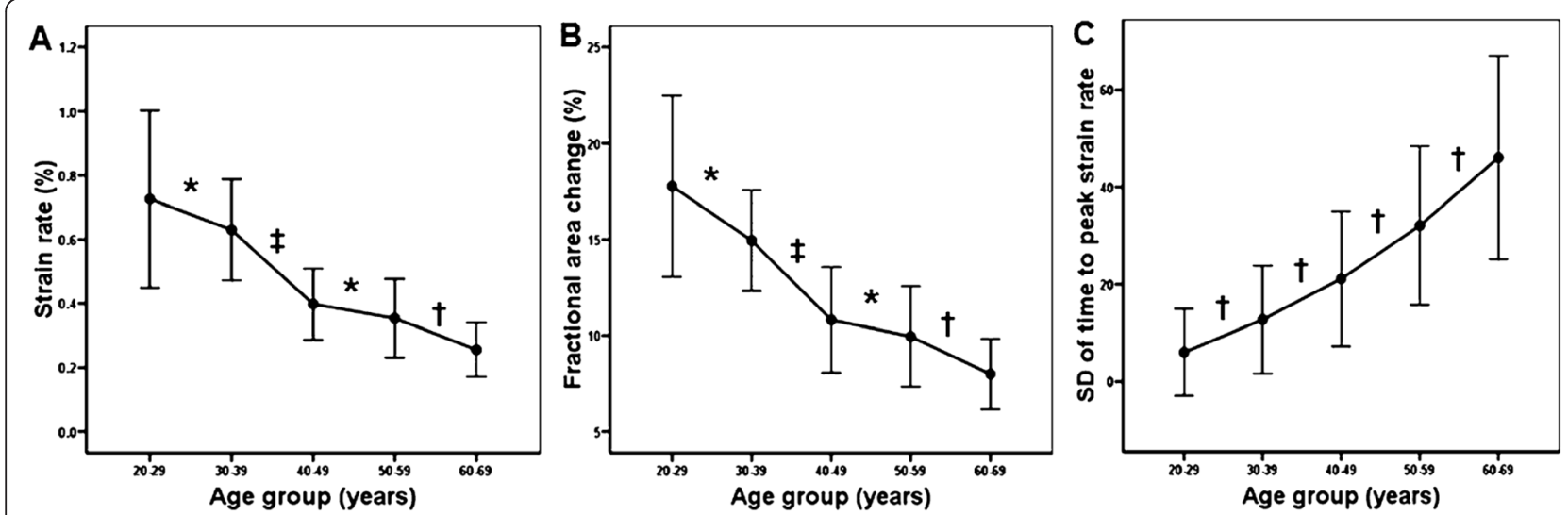

Fig. 3 a Average peak circumferential strain rate according to age group. b Fractional area change according to age group. c The SD of time to peak circumferential strain rate (Tsr-SD) according to age group. Data are presented as mean \pm SD. SD = standard deviation; Tsr $=$ time to peak circumferential strain rate. ${ }^{*} P>0.05,+P<0.05, \neq P<0.01$ (adapted from Ref [29]) 
detecting age-related vascular changes and determining cardiovascular risk [22, 23, 34].

However, up to the present, there are some limitations in evaluation of the arterial function with speckle tracking echocardiography. In cases of deep carotid arteries or compression by surrounding structures such as an enlarged thyroid or extended jugular vein, tracing of the carotid artery is technically difficult. Arterial wall is very thin compared to myocardium, so could be difficult to track. Even though, intra and interobserver variability were reliable in small studies, further studies with large subjects are necessary. The superiority of strain analysis compared with other methods of vascular functional assessment has not been confirmed yet. Longitudinal follow-up study with large populations needs to be evaluated to support the clinical usefulness of arterial strain analysis.

\section{Conclusions}

Recently, new ultrasound techniques have enabled the assessment of arterial mechanics using strain analysis. Several studies reported the feasibility of strain analysis in the evaluation of arterial function. The results of those studies demonstrated that strain analysis of superficial artery could be a valuable method to evaluate arterial function. If some technical problems in acquiring proper images and tracking thin arterial wall are improved, evaluation of arterial strain could be used extensively to assess local arterial function in future.

\section{Abbreviations \\ CVD: Cardiovascular disease; PWV: Pulse wave velocity; CIMT: Carotid intima- media thickness; CV: Cardiovascular; Wl: Velocity vector imaging; Alx: Augmentation index.}

\section{Competing interests}

The authors declare that they have no competing interests.

\section{Authors' contributions}

WIY summarized and the wrote manuscript, and HJW wrote and reviewed the manuscript. Both authors read and approved the final manuscript.

\section{Acknowledgements}

This work was supported by a Korea Science and Engineering Foundation (KOSEF) grant funded by the Korean government (M10642120001-06N421200110).

\footnotetext{
Author details

${ }^{1}$ Cardiology Division, CHA Bundang Medical Center, CHA University, Sungnam, Republic of Korea. ${ }^{2}$ Cardiology Division, Severance Cardiovascular Hospital, Yonsei University College of Medicine, Seoul, Republic of Korea. ${ }^{3}$ Severance Biomedical Science Institute, Yonsei University College of Medicine, 134 Shinchon-dongSeodaemun-gu, Seoul 120-752, Republic of Korea.
}

Received: 6 August 2015 Accepted: 10 November 2015

Published online: 16 December 2015

\section{References}

1. Gersh BJ, Sliwa K, Mayosi BM, Yusuf S. Novel therapeutic concepts: the epidemic of cardiovascular disease in the developing world: global implications. Eur Heart J. 2010;31(6):642-8.
2. Dzau VJ, Antman EM, Black HR, Hayes DL, Manson JE, Plutzky J, et al. The cardiovascular disease continuum validated: clinical evidence of improved patient outcomes: part II: Clinical trial evidence (acute coronary syndromes through renal disease) and future directions. Circulation. 2006;114(25):2871-91.

3. Dzau VJ, Antman EM, Black HR, Hayes DL, Manson JE, Plutzky J, et al. The cardiovascular disease continuum validated: clinical evidence of improved patient outcomes: part I: pathophysiology and clinical trial evidence (risk factors through stable coronary artery disease). Circulation. 2006;114(25):2850-70.

4. O'Rourke MF, Hashimoto J. Mechanical factors in arterial aging: a clinical perspective. J Am Coll Cardiol. 2007;50(1):1-13.

5. Yang EY, Chambless L, Sharrett AR, Virani S, Liu X, Tang Z, et al. Carotid arterial wall characteristics are associated with incident ischemic stroke but not coronary heart disease in the Atherosclerosis Risk in Communities (ARIC) study. Stroke. 2012;43(1):103-8.

6. Mattace-Raso FU, van der Cammen TJ, Hofman A, van Popele NM, Bos ML, Schalekamp MA, et al. Arterial stiffness and risk of coronary heart disease and stroke: the Rotterdam Study. Circulation. 2006;113(5):657-63.

7. Sutton Tyrrell K, Najjar S, Boudreau RM, Venkitachalam L, Kupelian V, Simonsick EM, et al. Elevated aortic pulse wave velocity, a marker of arterial stiffness, predicts cardiovascular events in well-functioning older adults. Circulation. 2005;111(25):3384-90.

8. O'Rourke MF, Safar ME, Dzau V. The cardiovascular continuum extended: aging effects on the aorta and microvasculature. Vasc Med. 2010;15(6):461-8.

9. Bank AJ, Wang H, Holte JE, Mullen K, Shammas R, Kubo SH. Contribution of collagen, elastin, and smooth muscle to in vivo human brachial artery wall stress and elastic modulus. Circulation. 1996;94(12):3263-70.

10. Virmani R, Avolio AP, Mergner WJ, Robinowitz M, Herderick EE, Cornhill JF, et al. Effect of aging on aortic morphology in populations with high and low prevalence of hypertension and atherosclerosis. Comparison between occidental and Chinese communities. Am J Pathol. 1991;139(5):1119-29.

11. Witteman JC, Grobbee DE, Valkenburg HA, van Hemert AM, Stijnen T, Burger $H$, et al. J-shaped relation between change in diastolic blood pressure and progression of aortic atherosclerosis. Lancet. 1994;343(8896):504-7.

12. Boutouyrie P, Bussy C, Lacolley P, Girerd X, Laloux B, Laurent S. Association between local pulse pressure, mean blood pressure, and large-artery remodeling. Circulation. 1999;100(13):1387-93.

13. Cheng GC, Loree HM, Kamm RD, Fishbein MC, Lee RT. Distribution of circumferential stress in ruptured and stable atherosclerotic lesions. A structural analysis with histopathological correlation. Circulation. 1993;87(4):1179-87.

14. Laurent S, Cockcroft J, Van Bortel L, Boutouyrie P, Giannattasio C, Hayoz D, et al. Expert consensus document on arterial stiffness: methodological issues and clinical applications. Eur Heart J. 2006;27(21):2588-605.

15. McEniery CM, Yasmin IR, Hall A, Qasem IB, Wilkinson JR, Cockcroft JR. Normal vascular aging: differential effects on wave reflection and aortic pulse wave velocity: the anglo-cardiff collaborative trial (ACCT). J Am Coll Cardiol. 2005;46(9):1753-60.

16. Chambless LE, Heiss G, Folsom AR, Rosamond W, Szklo M, Sharrett AR, et al. Association of coronary heart disease incidence with carotid arterial wall thickness and major risk factors: the atherosclerosis risk in communities (ARIC) study, 1987-1993. Am J Epidemiol. 1997;146(6):483-94.

17. O'Leary DH, Polak JF, Kronmal RA, Manolio TA, Burke GL, Wolfson Jr SK. Carotid-artery intima and media thickness as a risk factor for myocardial infarction and stroke in older adults. Cardiovascular health study collaborative research group. N Engl J Med. 1999;340(1):14-22.

18. Giannattasio C, Failla M, Capra A, Scanziani E, Amigoni M, Boffi L, et al. Increased arterial stiffness in normoglycemic normotensive offspring of type 2 diabetic parents. Hypertension. 2008;51(2):182-7.

19. Geyer H, Caracciolo G, Abe H, Wilansky S, Carerj S, Gentile F, et al. Assessment of myocardial mechanics using speckle tracking echocardiography: fundamentals and clinical applications. J Am Soc Echocardiogr. 2010;23(4):351-69.

20. Blessberger $H$, Binder T. NON-invasive imaging: Two dimensional speckle tracking echocardiography: basic principles. Heart. 2010;96(9):716-22.

21. Mondillo S, Galderisi M, Mele D, Cameli M, Lomoriello VS, Zac V, et al. Speckle-tracking echocardiography: a new technique for assessing myocardial function. J Ultrasound Med. 2011;3(1):71-83.

22. Bjllmark A, Lind B, Peolsson M, Shahgaldi K, Brodin L, Nowak J. Ultrasonographic strain imaging is superior to conventional non-invasive measures of vascular stiffness in the detection of age-dependent differences in 
the mechanical properties of the common carotid artery. Eur J Echocardiogr. 2010;11(7):630-6.

23. Catalano M, Lamberti Castronuovo A, Catalano A, Filocamo D, Zimbalatti C. Two-dimensional speckle-tracking strain imaging in the assessment of mechanical properties of carotid arteries: feasibility and comparison with conventional markers of subclinical atherosclerosis. Eur J Echocardiogr. 2011;12(7):528-35.

24. Kim SA, Park S, Kim M, Kim Y, Cho D, Ahn C, et al. The relationship between mechanical properties of carotid artery and coronary artery disease. Eur Heart J Cardiovasc Imaging. 2012;13(7):568-73.

25. Svedlund S, Eklund C, Robertsson P, Lomsky M, Gan L. Carotid artery longitudinal displacement predicts 1 -year cardiovascular outcome in patients with suspected coronary artery disease. Arterioscler Thromb Vasc Biol. 2011;31(7):1668-74.

26. Pirat B, McCulloch ML, Zoghbi WA. Evaluation of global and regional right ventricular systolic function in patients with pulmonary hypertension using a novel speckle tracking method. Am J Cardiol. 2006;98(5):699-704.

27. Cannesson M, Tanabe M, Suffoletto MS, Schwartzman D, Gorcsan J. Velocity vector imaging to quantify ventricular dyssynchrony and predict response to cardiac resynchronization therapy. Am J Cardiol. 2006;98(7):949-53.

28. Yang W, Shim C, Cho I, Chang H, Choi D, Jang Y, et al. Dyssynchronous systolic expansion of carotid artery in patients with marfan syndrome. J Am Soc Echocardiogr. 2010;23(12):1310-6.

29. Yang W, Shim CY, Bang WD, Oh CM, Chang HJ, Chung N, et al. Asynchronous arterial systolic expansion as a marker of vascular aging: assessment of the carotid artery with velocity vector imaging. J Hypertens. 2011;29(12):2404-12.

30. Cho IJ, Shim CY, Yang W, Kim S, Chang H, Jang Y, et al. Assessment of mechanical properties of common carotid artery in Takayasu's arteritis using velocity vector imaging. Cir J. 2010;74(7):1465-70.

31. Kim SA, Lee KH, Won HY, Park S, Chung JH, Jang Y, et al. Quantitative assessment of aortic elasticity with aging using velocity-vector imaging and its histologic correlation. Arterioscler Thromb Vasc Biol. 2013;33(6):1306-12.

32. Pannier BM, Avolio AP, Hoeks A, Mancia G, Takazawa K. Methods and devices for measuring arterial compliance in humans. Am J Hypertens. 2002;15(8):743-53.

33. Kanters SD, Elgersma OE, Banga JD, van Leeuwen MS, Algra A Reproducibility of measurements of intima-media thickness and distensibility in the common carotid artery. Eur J Vasc Endovasc Surg. 1998;16(1):28-35.

34. Kawasaki T, Fukuda S, Shimada K, Maeda K, Yoshida K, Sunada H, et al. Direct measurement of wall stiffness for carotid arteries by ultrasound strain imaging. J Am Soc Echocardiogr. 2009;22(12):1389-95.

\section{Submit your next manuscript to BioMed Central and take full advantage of:}

- Convenient online submission

- Thorough peer review

- No space constraints or color figure charges

- Immediate publication on acceptance

- Inclusion in PubMed, CAS, Scopus and Google Scholar

- Research which is freely available for redistribution 